\title{
Intradural Rupture of Lumbar Intervertebral Disc
}

\author{
Shih-Tseng Lee and David Fairholm
}

SUMMARY: Two cases of intradural disc rupture with cauda equina compression are reported. Myelography demonstrated complete block and at surgery intradural fragments of sequestrated disc material were found. The pathogenesis of this condition is reviewed and our recommendation for management is presented.

RÉSUMÉ: Nous rapportons deux cas de rupture intradurale de disques vertébraux avec compression de la queue de cheval. La myélographie démontra un bloc complet et à la chirurgie on trouva des fragments intraduraux du disque. Nous présentons une revue de la pathogénèse de cette condition et nos recommendations pour le traitement.

Can. J. Neurol. Sci. 1983; 10:192-194

Although disc herniation play an important role in low back pain, herniation of an extradural intervertebral disc through the dura mater into the subarachnoid space is rare. Dandy (1942), in a series of 300 cases with complications of ruptured intervertebral discs reported one case with L4-5 disc rupture into the subarachnoid space through the dura. Since then 41 cases have been reported in the literature (Dandy, 1942; Paterson \& Gray, 1952; Tucker, 1956; Lyons \& Wise, 1961; Wilson, 1962; Fisher, 1965; Robinson, 1965; Slater et al., 1965; Kulowski, 1967; Blikra, 1969; Peyser \& Harari, 1977; Hodge et al., 1978; Smith, 1961). The levels at which the disc rupture occurred in these 41 cases are summarized in Table 1. Most of those involving the lumbar level presented as a cauda equina syndrome and a myelographic block was almost always present.

\begin{tabular}{cc}
\hline Table 1: Level of occurrence of Intradural disc rupture In Itterature. \\
\hline \hline C5-6 & 1 \\
T9 & 1 \\
T10 & 1 \\
L1-2 & 5 \\
L2-3 & 5 \\
L3-4 & 10 \\
L4-5 & 17 \\
L5-S1 & 1 \\
Total & 41
\end{tabular}

From January 1980 through March 1982 a total of 179 cases of lumbar disectomy were done in this department. Among these there were two cases found to have fragments ruptured intradurally and they form the basis of this report.

\section{Case RePorts}

Case 1: This 66 year old male farmer presented with a 2 year history of low back pain radiating to both legs. Two months before admission he developed severe bilateral leg pain and progressive weakness with inability to walk, constipation, overflow dribbling of urine, and diminished pain, temperature, and touch sensation.

Examination on admission revealed marked spasm of the paraspinal muscles and marked bilateral limitation of straight leg raising with leg pain. He was unable to walk on the toes of either foot. There was moderate weakness of the ankle dorsiflexors bilaterally and mild weakness of both quadriceps. Both lower extremities showed moderate wasting with fasciculations. Pinprick, touch, temperature and vibration sense were reduced below the $\mathrm{L} 3$ level. The knee reflexes were diminished and ankle reflexes were absent. Anal sphincter tone was lax.

$\mathrm{X}$-rays of the lumbar spine showed marked degenerative changes at multiple levels and a myelogram showed a complete block at L3-4 with the characteristic appearance of an anterior extradural mass. (Fig. 1)

A laminectomy from L3 to L5 inclusive showed marked degenerative and hypertrophic changes. At the L3-4 level a large central disc herniation was found and radical removal was performed. A large mass was still palpable through the dura and on opening the dura, a $1.2 \times 1 \times 1.2 \mathrm{~cm}$ fragment of disc material was found. After separating the roots it was removed from its densly adherent attachment to the anterior dura. The roots were unaffected and the dura was closed. Examination in the early postoperative period showed him to be free of pain with marked improvement in his leg strength. On follow up 2 months later he had no leg pain and sensation was improved. Muscle strength was improved but had not returned to normal in all muscle groups. He could walk slowly without support. Sphincter function was only partially recovered.

Case 2: This 57 year old farmer with a long history of back pain was diagnosed as having a herniated disc and operated on 2 years previously at a local hospital. After operation he felt well for only 2 months before the back pain recurred accompanied by leg weakness. Two days before admission, associated with exertion, he had a sudden onset of severe back pain radiating to the right lower extremity. He noted numbness and decreased pain and temperature sensation over the right lower extremity. There were no bladder or bowel symptoms.

From the Division of Neurosurgery, Department of Surgery, Chang Gung Memorial Hospital, Taipei, Taiwan.

Received July 27, 1982. Accepted for publication March 8, 1983.

Reprint requests to: Dr. Shih-Tseng Lee, Chang Gung Memorial Hospital, 199 Tung Hwa North Road, Taipei, Taiwan 105. 


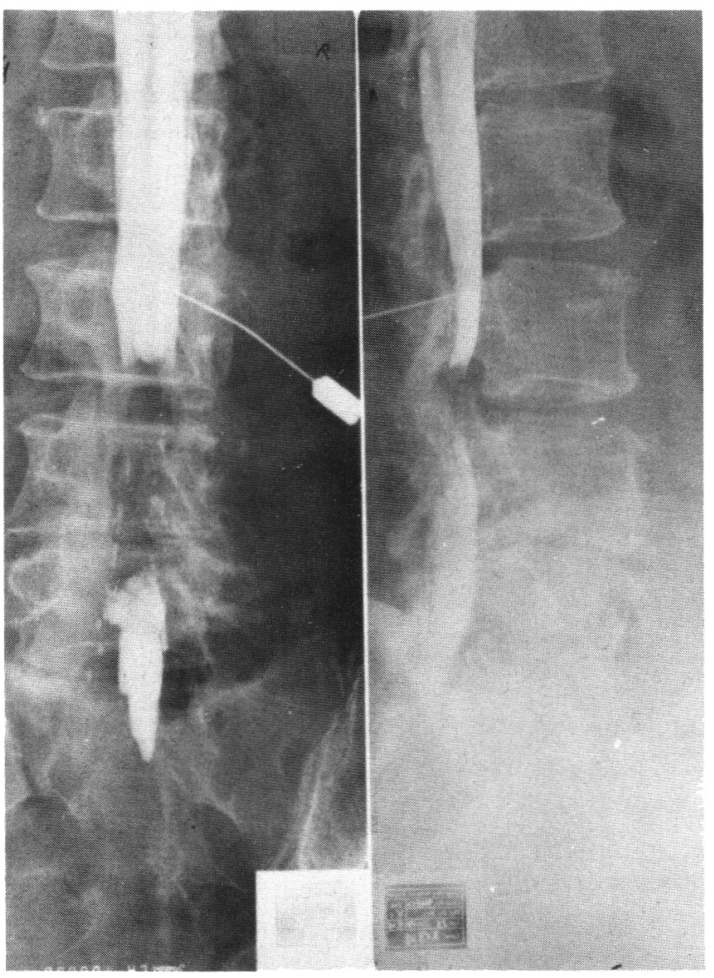

Figure l - (case 1) AP and lateral myelogram shows extensive degenerative change at multiple levels with complete block at L3-4.

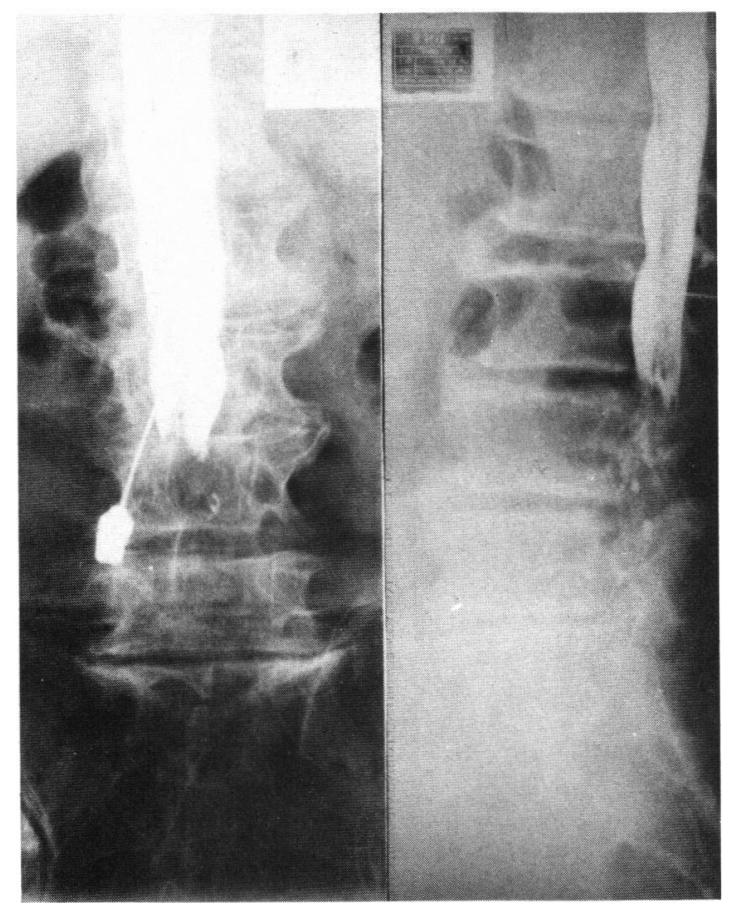

Figure $2-$ (case 2) AP and lateral myelogram shows narrowing at L4-5 and incomplete block at L3-4 due to anterior extradural compression.
Examination on admission revealed marked paraspinal spasm with scoliosis and marked limitation of straight leg raising with bilateral leg pain. He was unable to walk on the toes on either side and there was moderate weakness of the ankle dorsiflexors bilaterally, more on the left side. There was decreased pain, touch, and temperature sensation over the L4 and L5 distribution bilaterally. The left knee reflex and both ankle reflexes were absent. The sphincters were normal. X-rays of the lumbar spine showed moderate degenerative changes with L4-5 disc space narrowing. A myelogram showed obstruction at L3-4 with an anterior extradural mass compression. (Fig. 2)

A laminectomy from L3 to L5 inclusive revealed moderate hypertrophic and degenerative changes. The ligamentum flavum and dura were tightly adherent at the L4-5 level. After separation of the adhesions, a large central sequestrated fragment of disc material was found compressing the L5 roots bilaterally. After removal of the sequestrated fragment, a radical removal of the L4-5 disc was performed. A large mass was still palpable through the dura and on opening the dura a $1 \times 1 \times 2 \mathrm{~cm}$ fragment of disc material was found compressing the cauda equina. After separating the adherance to roots it was removed from its densely adherent attachment to the anterior dura. The dura was closed with a graft.

Examination over the next several days showed him to be free of pain with marked improvement in his leg strength. Two months later he had no leg pain and his strength was markedly improved. He could walk on his toes without support. Power in the ankle dorsiflexors, sensation, and sphincter function were all normal.

\section{Discussion}

The intervertebral disc is composed of cartilagenous end plates, nucleus pulposus, fibracartilage, and an annulus fibrosus which is strengthened by anterior and posterior longitudinal ligaments. Primary chemical changes within disc material result in decreased water content (Rothman, 1972) allowing excess movement and rotation to occur which causes weakening and cracking in the hyaline cartilage plate. Despite the posterior central strengthening by the posterior longitudinal ligament, a significant number of discs protrude in the midline. Although small laterally projecting discs can cause unilateral root compression, central protrusions are usually large before producing symptoms and commonly affect roots bilaterally or cause a cauda equina compression. Some discs are protruded with sufficient force to be entirely sequestrated. If these are central, the whole force and pressure is brought to bear on the central anterior dura. Sequestrated discs are usually irregular and may have sharp protruding edges. Should the force be excessive or the pressure maintained over a long period of time, then the weakened dura may be penetrated by the large sequestrated fragment. When the dura is penetrated the decreased resistance allows the fragment to migrate within the subarachnoid space.

Blikra (1969) reported a study of 40 post-mortem cases which had never suffered from back pain or sciatica. He found at the L4-5 interspace a firm connection between the dura, the posterior longitudinal ligament, and the annulus that could not be divided by blunt dissection. At L5-S1, and L3-4 some connection was also found but could be separated easily. Peyser (1977) proposed that previous adhesions between the ventral dura mater and posterior longitudinal ligament are possibly due to an inflammatory process or previous surgery. In the 41 reported 
cases, about one third had had previous disc surgery, confirming Peyser's proposal.

Penning (1981) in a recent study on biomechanics of the lumbosacral dural sac using flexion-extension myelography, found that dural sac changes are variable at different levels. Most stretch occurs between L4 and L5, which over years may lead to weakening of the dura at this level. With a history of previous inflammatory disease or surgery causing adhesions, a new, large, or sequestrated disc fragment has a greater chance of penetraing the dura and entering the subarachnoid space at this level.

In our two cases dense adhesions which could not easily be separated were found between the dura, posterior longitudinal ligament, and annulus. Previous disc surgery at the same level was responsible in one case. As reported in the literature, both of our cases had prolonged low back pain and a long course which did not respond to conservative treatment. Likewise both had blocks on myelogram and at surgery dense adhesions between the anterior dura and posterior longitudinal ligament were found. The sequestrated intradural disc fragments were adherent to the anterior dura and nerve roots. Complete removal allowed good recovery following surgery.

\section{REFERENCES}

Blikra, G. (1969). Intradural herniated lumbar disc. J. Neurosurg. 31:676-679.

Dandy, W.E. (1942). Serious complications of ruptured intervertebral disks. Jama 119:474-477.
Fisher, R.G. (1965). Protrusions of thoracic disc. The factor of herniation through the dura mater. J. Neurosurg. 22:591-593.

Hodge, C.J., Binet, E.F., Kieffer, S.A. (1978). Intradural herniation of lumbar intervertebral discs. Spine 3:346-350.

Kulowski, J. (1967). Subdural rupture of an intervertebral lumbar disc. Mo. Med. 64:715-716.

Lyons, A.E. \& Wise, B.L. (1961). Subarachnoid rupture of intervertebral disc fragments. J. Neurosurg. 18:242-244.

Paterson, J.E. \& Gray, W. (1952). Herniated nucleus pulposus: The free fragment. Br. J. Surg. 39:509-513.

Penning, L. \& Wilmink, J.T. (1981). Biomechanics of lumbosacral dural sac: A study of flexion-extension myelography. Spine 6, 4:398-408.

Peyser, E. \& Harari, A. (1977). Intradural rupture of lumbar intervertebral disk: Report of two cases with review of the literature. Surg. Neurol. 8:95-98.

Robinson, R. G. (1965). Massive protusions of lumbar disks. Br. J. Surg. 52:858-865

Rothman, R.H. (1972). The pathophysiology of disc degeneration. Clinical Neurosurg. 20:174-182.

Slater, R.A., Pineda, A. \& Porter, R.W. (1965). Intradural herniation of lumbar intervertebral discs. Arch. Surg. 90:266-269.

Smith, R.V. (1981). Intradural disc rupture: Report of two cases. J. Neurosurg. 55:117-120.

Tucker, A.S. (1956). Myelography of complete spinal obstruction. Am. J. Roentgenol. 76:248-269.

Wilson, P.J.E. (1962). Cauda equina compression due to intrathecal herniation of an intervertebral disk: A case report. Br. J. Surg. 49:423-428. 